\title{
Oral contraceptive use and the clinical course of Crohn's disease: a prospective cohort study
}

\author{
J Cosnes, F Carbonnel, F Carrat, L Beaugerie, J-P Gendre
}

\begin{abstract}
Background-Women with Crohn's disease are usually advised not to take oral contraceptives, but, unlike smoking, there is no clear association between current oral contraceptive use and more severe disease.

Aim-To assess the effect of oral contraceptive use on the clinical course of Crohn's disease.

Patients-331 women, aged 16-50 years, with Crohn's disease and Crohn's disease activity index $<200$, were enrolled consecutively during a one year period. Patients were classified at inclusion as oral contraceptive users or non-users and smokers or non-smokers.

Methods-A prospective 12-18 month cohort study was used. The main outcome measures were flare up rate and time to flare up.

Results-In total, 134 women used oral contraceptives, in most cases low oestrogen formulations. During the study period, 61 oral contraceptive users $(46 \%)$ developed a flare up, compared with 85 non-users $(43 \%)$. The hazard ratio for oral contraceptive use was 1.11 (95\% confidence interval 0.80 to 1.55$)$. Variables associated with flare up were smoking status, recently active disease, baseline Crohn's disease activity index, and presence of anoperineal lesions. The same results were obtained when the analysis was restricted to patients eligible for a relapse prevention trial.

Conclusion-Unlike smoking, oral contraceptives have no effect on Crohn's disease activity.

(Gut 1999;45:218-222)

Keywords: Crohn's disease; smoking; oral contraceptives
\end{abstract}

Service logie et Nutrition, Hôpital Rothschild, Paris, France

J Cosnes

F Carbonnel

L Beaugerie

J-P Gendre

Unité de Biostatistique et d'Informatique Médicale, Hôpital Saint-Antoine, Paris

F Carrat

Correspondence to: Professor J Cosnes, Hôpital Rothschild, $33 \mathrm{Bd}$ de Picpus, 75571 Paris cedex 12,

France.

Accepted for publication 17 February 1999
Smoking both influences the development of Crohn's disease ${ }^{1}$ and affects the disease course. $^{2-6}$ In particular, patients with Crohn's disease who smoke have been reported to experience significantly more relapses, ${ }^{3}$ have more operations, ${ }^{45}$ and need steroids and immunosuppressants more often ${ }^{6}$ than nonsmokers with Crohn's disease.

The main mechanism by which current smoking potentially exacerbates Crohn's disease may be the worsening of vascular intestinal lesions, as multifocal infarction is recognised as having an important pathogenic role in Crohn's disease. ${ }^{7}$ Additional evidence for Crohn's disease having a vascular aetio- pathogeny is the infrequent occurrence of inflammatory bowel disease in patients with haemophilia and von Willebrand disease. ${ }^{8}$

The observation that the effect of smoking on Crohn's disease relapse ${ }^{46}$ was more pronounced in women led to the speculation that oral contraceptive use, by enhancing a tendency to focal thrombosis, ${ }^{9}$ may be a confounding factor, or have an additive effect to that of smoking. Indeed, oral contraceptives have been suggested to worsen the clinical course of Crohn's disease. ${ }^{10}$ Actually, the evidence for a potentiating effect of the oral contraceptive pill on Crohn's disease is limited, ${ }^{11} 12$ although several studies have reported a weak but consistent association between oral contraceptive use and the occurrence of Crohn's disease. ${ }^{13-15}$ The studies that focused on the effect of oral contraceptives on the course of the disease ${ }^{16-18}$ included different groups of selected patients, examined various end points, and gave conflicting results.

To evaluate the effect of oral contraceptive use on Crohn's disease activity, we conducted a prospective study in a large cohort of consecutive patients followed in a single centre.

\section{Methods}

All female patients with Crohn's disease attending our outpatient unit from February 1995 to January 1996 were evaluated for inclusion in the study. The inclusion criteria were an established diagnosis of Crohn's disease, ${ }^{19}$ age of 16 to 50 years, and little or no disease activity for one week before inclusion, as defined by a Crohn's disease activity index (CDAI) value ${ }^{20}$ below 200 .

\section{STUDY DESIGN}

The flare up rate and the time to flare up over a period of 12-18 months were compared in two groups of patients, oral contraceptive users and non-users, defined by oral contraceptive use at baseline. At inclusion, patients had a clinical examination, their packed cell volume was determined, and their CDAI was calculated. They were then followed up regularly by their usual doctor in our unit, who was not blinded to the oral contraceptive use or smoking status of the patient. Visits were scheduled every three to six months and patients were given instructions to telephone us if there were new symptoms. In the event of a flare up, patients were seen immediately, and CDAI was calculated retrospectively for the preceding week. The study period ended with the first visit after

Abbreviation used in this paper: CDAI, Crohn's disease activity index. 
Table 1 Baseline characteristics of patients with Crohn's disease related to oral contraceptive use

\begin{tabular}{llll}
\hline Characteristic & $\begin{array}{l}\text { Non-users } \\
(n=197)\end{array}$ & $\begin{array}{l}\text { Users } \\
(n=134)\end{array}$ & p Value \\
\hline Age (years) & $32(27-40)$ & $28(25-33)$ & 0.00001 \\
Duration of disease (months) & $78(38-136)$ & $52(22-99)$ & 0.0005 \\
Crohn's disease activity index & $80(52-118)$ & $76(49-122)$ & NS \\
Active disease during the previous year & $111(56)$ & $89(66)$ & NS \\
$\begin{array}{l}\text { Active intestinal lesions } \\
\text { Current smokers }\end{array}$ & $163(83)$ & $110(82)$ & NS \\
$\begin{array}{l}\text { Current therapy } \\
\quad \text { Mesalamine, olsalazine or sulphasalazine }\end{array}$ & $108(55)$ & $78(58)$ & NS \\
$\quad$ Steroids & $106(54)$ & $68(51)$ & NS \\
$\quad$ Azathioprine or methotrexate & $58(29)$ & $42(31)$ & NS \\
$\quad$ No treatment & $50(25)$ & $29(22)$ & NS \\
\hline
\end{tabular}

Results are expressed as median (interquartile range) or number (\%).

12 months of follow up, then its duration varied from 12 to 18 months. Forty patients $(12 \%$ in each group) referred from another centre during the period of inclusion were followed up elsewhere. Data were collected in these patients by contacting their personal doctor.

\section{CHARACTERISTICS AT INCLUSION}

Patients were asked about their current use of contraceptive pills and classified as oral contraceptive users if they were taking the pill during the month before inclusion. They were also asked about smoking habits, and classified as current smokers if they had smoked more than seven cigarettes a week for at least six months ${ }^{46}$ and had reported any cigarette smoking in the previous six months. Characteristics of Crohn's disease were recorded. The disease was considered as recently active if the patient had had a flare up episode, chronic active disease, or anoperineal complications during the year preceding inclusion.

\section{TREATMENT POLICY}

As maintenance treatment, we used aminosalicylate derivatives (sulphasalazine, olsalazine, or mesalamine, 2-3 g daily) for asymptomatic or moderately active forms of the disease, and immunosuppressive drugs (azathioprine 2 $\mathrm{mg} / \mathrm{kg}$ a day or intramuscular methotrexate 10-20 mg a week) for severe forms. Flare up episodes were treated with mesalamine (3-4 g daily) or prednisolone ( $1 \mathrm{mg} / \mathrm{kg}$ a day, progressively tapered after four weeks), according to their clinical severity. When steroid therapy failed, patients were given a four week course of enteral or parenteral nutrition. Surgery was reserved for stenotic and extraparietal complications or intractable forms.

\section{EVALUATION CRITERIA}

The outcome was the flare up rate and the time to flare up. Flare up was defined as an increase in the CDAI of at least 60 greater than baseline and exceeding 150. In patients with chronic perianal disease, flare up was defined as the development of an abscess or a new fistula. Additional measures of evaluation included the need for surgery, for stronger medical treatment (steroid course, nutritional support, introduction of immunosuppressants), for hospitalisation, and the number of working days lost due to sickness.
STATISTICAL ANALYSIS

Continuous data are expressed as median (interquartile range), and differences between the two groups were tested for significance by Wilcoxon test. Discrete data are given as percentages, and comparisons were made by the Pearson $\chi^{2}$ test. Kaplan-Meier survival analysis was used to estimate the cumulative probability of developing a flare up episode in the 12-18 months after inclusion. Survival curves were compared by means of a two sided log rank test. A Cox proportional hazards regression model with a backward variable elimination procedure was used to assess the strength of the associations while controlling for possible confounding variables. All baseline variables suspected to be possible predictors of flare up (age, duration of disease, recently active disease, CDAI value $(<$ or $>150)$, small bowel lesions, colonic lesions, anoperineal lesions, 5-aminosalicylate, steroid or immunosuppressive therapy, smoking status, and oral contraceptive use) were entered into the model. Interaction between oral contraceptive use and smoking was tested. A further similar analysis estimating the cumulative probability of relapse was performed in a subgroup of patients whose disease was in remission at baseline. Results of analysis are presented as hazard ratios with $95 \%$ confidence intervals. Calculations were performed using Statview statistical software.

\section{Results}

A total of 334 patients were considered for inclusion in the study. Of these, 16 with CDAI of 200 or more and nine who required urgent hospitalisation received appropriate treatment and were included after their CDAI had decreased to under 200. Three patients were excluded: two were only seen during a flare up episode and did not come back thereafter; one was living abroad and was unable to maintain further contact. Fourteen women used a progesterone-only pill, 115 used a 30 or $35 \mu \mathrm{g}$ oestrogen formulation, and five used a $50 \mu \mathrm{g}$ oestrogen formulation. In all, 197 women were not taking the pill at inclusion; of these, seven were pregnant. Table 1 gives the baseline characteristics of the two groups. Compared with non-users, oral contraceptive users were younger and had a disease of shorter duration. A larger proportion of them had active colonic lesions $(59 \% v 47 \%$ in non-users, $\mathrm{p}=0.03)$.

\section{OUTCOME}

One patient, a 39 year old heavy smoker, having a chronic active disease poorly controlled with methotrexate, died during the study period. Death occurred suddenly at home, while she was starting a tobacco weaning programme. She was not taking oral contraceptives. No autopsy was performed. During the study period, we did not observe any case of arterial or venous thrombosis, but all inpatients were given prophylactic anticoagulant treatment with low molecular mass heparin. Five oral contraceptive users who stopped taking the pill and 11 non-users became pregnant after inclusion. Figure 1 gives the actuarial rate 


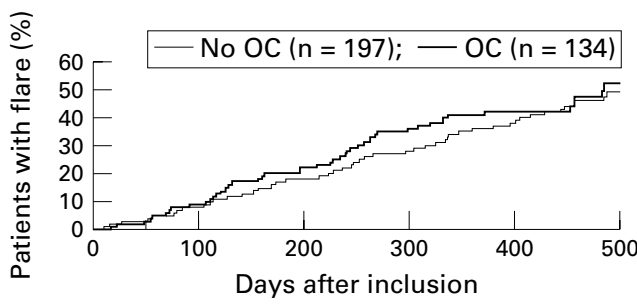

Figure 1 Kaplan-Meier life table analysis of the occurrence of a flare up episode of Crohn's disease in the study population, according to oral contraceptive (OC) use at baseline.

of flare up episodes in the two groups. Median (SD) time to flare up was 484 (30) days in oral contraceptive users compared with 504 (41) days in non-users. The two curves were not significantly different. The flare up rate was not different according to the nature of the oral contraceptive (29\% for progesterone-only oral contraceptives, and 40 and $46 \%$ for high and low dose combined oral contraceptives respectively). Median CDAI during flare up was not different in oral contraceptive users and non-users (246 $v 241$ respectively). The flare up was controlled by increasing mesalamine dosage or a steroid course in 40 oral contraceptive users $(66 \%)$ and 64 non-users $(75 \%$, difference not significant). Table 2 gives other therapeutic needs and events that occurred during the study period in the two groups. Hospitalisation rate was similar in the two

Table 2 Main events during the study period

\begin{tabular}{llll}
\hline & Non-users $(n=197)$ & Users $(n=134)$ & p Value \\
\hline Deceased & 1 & 0 & NS \\
Lost to follow up & 2 & 1 & NS \\
Duration of study period (days) & $397(371-481)$ & $412(379-462)$ & NS \\
Flare up episode & $85(43)$ & $61(46)$ & NS \\
Steroid course & $40(20)$ & $30(22)$ & NS \\
Enteral or parenteral nutrition course & $11(6)$ & $5(4)$ & NS \\
Introduction of immunosuppressants & $20(10)$ & $11(8)$ & NS \\
Excisional surgery & $15(8)$ & $9(7)$ & NS \\
Anoperineal surgery & $6(3)$ & $9(7)$ & NS \\
Patients requiring hospitalisation & $58(30)$ & $38(29)$ & NS \\
$>3$ days of work lost & $42(30)$ & $43(41)$ & 0.08 \\
\hline
\end{tabular}

Results are expressed as median (interquartile range) or number (\%).

$\star$ Percentage calculated for patients at work at baseline.

Table 3 Crude flare up rates during the study period according to baseline variables

\begin{tabular}{|c|c|c|c|}
\hline & $\begin{array}{l}\text { No (\%) of patients with } \\
\text { flare up }\end{array}$ & $\begin{array}{l}\text { Median time to } \\
\text { flare up (days) }\end{array}$ & $\begin{array}{l}\text { p Value } \\
\text { (log rank test) }\end{array}$ \\
\hline Total & $146 / 328(45)$ & $504(49)$ & \\
\hline \multicolumn{4}{|c|}{ Recently active disease } \\
\hline Yes & $107 / 180(59)$ & $337(7)$ & \multirow[t]{2}{*}{$<0.0001$} \\
\hline No & $39 / 148(26)$ & $>614$ & \\
\hline \multicolumn{4}{|l|}{ CDAI } \\
\hline$>150$ & $36 / 49(73)$ & $263(89)$ & \multirow{2}{*}{$<0.0001$} \\
\hline$<151$ & $110 / 279(39)$ & $548(18)$ & \\
\hline \multicolumn{4}{|c|}{ Colonic lesions } \\
\hline Yes & $91 / 169(54)$ & $405(26)$ & \multirow[t]{2}{*}{0.0004} \\
\hline No & $55 / 159(35)$ & $>614$ & \\
\hline \multicolumn{4}{|c|}{ Anoperineal lesions } \\
\hline Yes & $41 / 68(60)$ & $266(62)$ & \multirow[t]{2}{*}{$<0.0001$} \\
\hline No & $105 / 260(40)$ & $535(14)$ & \\
\hline \multicolumn{4}{|c|}{ Steroid therapy } \\
\hline Yes & $65 / 100(65)$ & $355(8)$ & \multirow[t]{2}{*}{$<0.0001$} \\
\hline No & $81 / 228(35)$ & $>614$ & \\
\hline \multicolumn{4}{|c|}{ Current smoking } \\
\hline Yes & $97 / 183(53)$ & $444(29)$ & \multirow[t]{2}{*}{0.002} \\
\hline No & $49 / 145(34)$ & $>581$ & \\
\hline
\end{tabular}

Results for time to flare up are expressed as median (SD).

Note: The following were not significant: age, duration of disease, small bowel lesions, previous intestinal resection, 5-aminosalicylate treatment, immunosuppressive therapy, use of oral contraceptives.

CDAI, Crohn's disease activity index.
Table 4 Predictors of flare up according to multivariate Cox analysis

\begin{tabular}{llll}
\hline & $\begin{array}{l}\text { Adjusted } \\
\text { hazard ratio }\end{array}$ & $95 \%$ CI & p Value \\
\hline Recently active disease & 2.17 & 1.47 to 3.22 & 0.0001 \\
CDAI $>150$ & 1.94 & 1.30 to 2.90 & 0.0012 \\
Anoperineal lesions & 1.92 & 1.32 to 2.79 & 0.0006 \\
Current smoking & 1.54 & 1.09 to 2.18 & 0.0152 \\
\hline CDAI, Crohn's disease activity index; CI, confidence interval.
\end{tabular}

groups, with length of stay exceeding five days in 17 users $(13 \%)$ compared with 36 non-users (18\%, difference not significant).

PREDICTION OF FLARE UP AND RELAPSE

Univariate analysis by life table methods showed significantly different flare up rates depending on recently active disease, CDAI, colonic lesions, anoperineal lesions, steroid therapy, and smoking status (table 3). Oral contraceptive use had no effect (hazard ratio $1.11 ; 95 \%$ confidence interval 0.80 to 1.55$)$. A regression model selected four variables: recently active disease, CDAI, anoperineal lesions, and smoking status (table 4). No significant interaction was found between smoking and oral contraceptive use.

When the analysis was restricted to the 158 patients who would have been eligible for a relapse prevention trial-that is, CDAI $<150$, no recent steroid or immunosuppressive therapy, no chronic perianal disease-results were similar: the use of oral contraceptives was not associated with an increased relapse rate (hazard ratio $1.26 ; 95 \%$ confidence interval 0.71 to 2.23 ), and multivariate analysis retained as predictors a recent disease activity, anoperineal lesions, and smoking status. Current smoking was associated with a nearly threefold risk of relapse (adjusted hazard ratio 2.84; $95 \%$ confidence interval 1.53 to 5.25 ). There was no statistical evidence for interaction between oral contraceptives and smoking.

\section{Discussion}

This prospective study of a large cohort of women with Crohn's disease, while confirming the harmful effect of smoking, showed that the current use of oral contraceptives had no effect on activity of the disease.

Because the effect of environmental factors may be only apparent in certain subgroups of patients with Crohn's disease, care was taken in this study to include all the consecutive patients seen during a long enrolment period of a year. We believe that this mode of recruitment minimised selection bias and allowed us to obtain an accurate estimation of the real effects of environmental factors. Indeed, the deleterious effect of smoking was evident. Regarding this result, however, it should be noted that, for practical reasons, in this study the same doctor included and then followed an individual patient. Thus he was not blinded to the smoking status or the oral contraceptive use of the patient. There is, however, no obvious reason why this might have influenced recognition of a flare up or important therapeutic decisions such as steroid treatment, introduction of immunosuppressants, or surgical resection. 
Besides, when considering the effect of oral contraceptives in particular, some bias may be of concern. ${ }^{11}$ Patients with recently active disease may be more likely to take oral contraceptives than those with quiescent disease, in order to prevent a pregnancy which might affect their clinical status. Moreover, it has been suggested that smokers are more likely to take oral contraceptives than non-smokers, ${ }^{13}$ and that oral contraceptive users are more likely to smoke. ${ }^{14}$ In fact, in the present study, the proportions of oral contraceptive users did not differ significantly, whether or not Crohn's disease had been active during the year preceding inclusion, and were similar among smokers and non-smokers.

The results of this study are discordant with those of Timmer et $a l^{18}$ who reported an increased risk of relapse in patients receiving oral contraceptives. Several differences in the design of the two studies may explain the discrepancies. Firstly, the Canadian study included only patients who participated in a controlled trial of mesalamine for remission of Crohn's disease and were placebo treated. At the start of the study, all patients were in remission, had no chronic perianal disease, and had not used steroids or immunosuppressants for several weeks. When selecting from our patients those fulfilling such criteria, we found, however, no difference in relapse rates between oral contraceptive users and non-users. Secondly, in the Canadian study, previous and current oral contraceptive users were combined into an "ever used oral contraceptives" group to avoid insufficient stratum sizes. The risk of relapse was higher in previous users, and when compared separately with those who had never used oral contraceptives, current users did not have a significantly different relapse rate. Finally, Timmer et $a l^{18}$ did not gather information on the nature and strength of the oral contraceptive used, but hypothesised that former users were more likely to have taken high dose, and current users low dose oral contraceptives. It has been suggested that a threshold dosage of oestrogen may be required to promote thromboembolic events. ${ }^{9} 1021$ The current trend towards prescribing low dose oral contraceptives may also explain why more recent studies failed to find an association between oral contraceptives and the occurrence of Crohn's disease. ${ }^{11}{ }^{12}$ In our study, the absence of a significant effect of current use of oral contraceptives was probably linked to the low oestrogen dosage of most of the pills used. The power of the study was $80 \%$ to detect hazard ratios $>2$ for a baseline hazard rate of $40 \%$. Although smoking did increase the flare up rate, the use of oral contraceptives did not potentiate the effect of smoking and there was no interaction between the two exposures. Other studies also failed to show any significant impact of oral contraceptives on the course of Crohn's disease: Wright ${ }^{16}$ found a trend for oral contraceptive users to have ileocolitis and more inflammatory attacks, but the first relapse rate was not significantly different between oral contraceptive users and non-users. Sutherland et $a l^{17}$ compared the risk of recurrence after surgery according to the use of oral contraceptives during the first year after surgery and showed that the five year actuarial rate of additional surgery was not statistically different: $25 \%$ for oral contraceptive users compared with $28 \%$ for non-users. Thus it is clear that low dose oral contraceptive use is not associated with a more severe evolution of Crohn's disease.

Thromboembolic disease is a significant cause of morbidity and mortality in patients with inflammatory bowel disease. Although we did not observe any thrombotic event during the period of study, caution must be observed with regard to the use of oral contraceptives in certain subgroups of patients, specifically those with acute exacerbations of their disease, ${ }^{22}$ those with inherited causes of thrombophilia, ${ }^{23}$ and finally smokers, in view of the striking synergy between oral contraceptives, including low oestrogen formulations, and smoking as regards the risk of haemorrhagic stroke ${ }^{24}$ and myocardial infarction. ${ }^{25}$ Patients with Crohn's disease who smoke must be encouraged to stop smoking, and it is essential to provide them with easy access to smoking cessation programmes. However, there is no reason connected with Crohn's disease activity to recommend methods of contraception other than low oestrogen oral contraceptives.

We are indebted to the following doctors for their clinical care of study patients and for assistance in collecting the data: Hélène d'Almagne-Serrano, Philippe Baumer, Jérôme Bellanger, JeanFrançois Contou, Daniel Evard, Philippe Godeberge, Philippe Lamy, Yves Le Quintrec, and Yann Ngô

1 Calkins BM. A meta-analysis of the role of smoking in inflammatory bowel disease. Dig Dis Sci 1989;34:1841-54. 2 Lindberg E, Jarnerot G, Huitfeldt B. Smoking in Crohn's disease: effect on localisation and clinical course. Gut 1992;33:779-82.

3 Duffy LC, Zielezny MA, Marshall JR, et al. Cigarette smoking and risk of clinical relapse in patients with Crohn's disease. Am F Prev Med 1990;6:161-6.

4 Sutherland LR, Ramcharan S, Bryant H, et al. Effect of cigarette smoking on recurrence of Crohn's disease. Gastroenterology 1990;98:1123-8.

5 Cottone M, Rosselli M, Orlando A, et al. Smoking habits and recurrence in Crohn's disease. Gastroenterology 1994; 106:643-48.

6 Cosnes J, Carbonnel F, Beaugerie L, et al. Effects of smoking on the long term course of Crohn's disease. Gastroentering on the long term cour

7 Wakefield AJ, Sawyerr AM, Dhillon AP, et al. Pathogenesis of Crohn's disease: multifocal gastrointestinal infarction. Lancet 1989;1:1057-62.

8 Thompson NP, Wakefield AJ, Pounder RE. Inherited disorders of coagulation appear to protect against inflammatory bowel disease. Gastroenterology 1995;108:1011-15.

9 Beller FK, Ehert C. Effects of oral contraceptives on blood coagulation. A review. Obstet Gynecol Surv 1988;40:42536.

10 Wakefield AJ, Sawyerr AM, Hudson M, et al. Smoking, the oral contraceptive pill, and Crohn's disease. Dig Dis Sci 1991;36:1147-50.

11 Lashner BA, Kane SV, Hanauer SB. Lack of association between oral contraceptive use and Crohn's disease: a community-based matched case-control study. Gastroenterology 1989;97:1442-7.

12 Godet PG, May GR, Sutherland LR. Meta-analysis of the role of oral contraceptive agents in inflammatory bowel role of oral contraceptive agent
disease. Gut 1995;37:668-73

13 Katschinski B, Fingerle D, Scherbaum B, et al. Oral contraceptive use and cigarette smoking in Crohn's disease. Dig Dis Sci 1993;38:1596-600

14 Sandler RS, Wurzelmann JL, Lyles CM. Oral contraceptive use and the risk of inflammatory bowel disease. Epidemiology 1992;3:374-8.

15 Boyko EJ, Theis MK, Vaughan TL, Nicol-Blades B. Increased risk of inflammatory bowel disease associated with oral contraceptive use. Am F Epidemiol 1994;140:26878

16 Wright JP. Factors influencing first relapse in patients with Crohn's disease. F Clin Gastroenterol 1992;15:12-16.

17 Sutherland LR, Ramcharan S, Bryant H, et al. Effect of oral contraceptive use on reoperation following surgery for contraceptive use on reoperation following
Crohn's disease. Dig Dis Sci 1992;37:1377-82.

18 Timmer A, Sutherland LR, Martin F, et al. Oral contraceptive use and smoking are risk factors for relapse in Crohn's disease. Gastroenterology 1998;114:1143-50. 
19 Lennard-Jones JE. Classification of inflammatory bowel disease. Scand $\mathcal{F}$ Gastroenterol 1989;24:2-6.

20 Best WR, Becktel JM, Singleton JW. Rederived values of eight coefficients of the Crohn's disease activity index CDAI). Gastroenterology 1979;77:843-6.

21 Poller L. Oral contraceptives, blood clotting and thrombosis. Br Med Bull 1978;34:151-6.

22 Talbot RW, Heppell J, Dozois RR, et al. Vascular complications of inflammatory bowel disease. Mayo Clin Proc 1986; 61:140-4.
23 Liebman HA, Kashani N, Sutherland D, et al. The factor V Leiden mutation increases the risk of venous thrombosis in patients with inflammatory bowel disease. Gastroenterology 1998;115:830-4

24 Petitti DB, Sidney S, Bernstein A, et al. Stroke in users of low-dose oral contraceptives. N Engl f Med 1996;335:815

25 Hennekens $\mathrm{CH}$, MacMahon B. Oral contraceptives and myocardal infarction. N Engl f Med 1977;296:1166-7. 